\title{
EXISTENCE OF TIME-SCALE CLASS OF THREE DIMENSIONAL FRACTIONAL DIFFERENTIAL EQUATIONS
}

\section{RABHA W. IBRAHIM ${ }^{1, *}$, AND MASLINA DARUS ${ }^{2}$}

\author{
${ }^{1}$ IEEE:94086547 \\ ${ }^{2}$ Centre of Modelling and Data Science, Faculty of Sciences and Technology, \\ Universiti Kebangsaan Malaysia,43600 Bangi, Selangor, Malaysia; maslina@ukm.edu.my
}

${ }^{*}$ Corresponding author: rabhaibrahim@yahoo.com

Abstract. The holomorphic results for fractional differential operator formals have been established. The analytic continuation of these outcomes has been studied for the fractional differential formal

$$
\left\{\begin{array}{l}
\frac{\partial^{\alpha} v(\wp, z)}{\partial \wp^{\alpha}}=\mathfrak{H}\left(\wp, z, v, \frac{\partial v}{\partial z}, \frac{\partial^{2} v}{\partial z^{2}}\right), \quad \alpha \in[0,1) \\
v(a, z)=\psi(z), \text { in a proximity to } z \in U,
\end{array}\right.
$$

where $U$ is the open unit disk. The benefit of such a problem is that a generalization of two significant problems: the Cauchy problem and the diffusion problem. Moreover, the analytic solution is given inside the open unit disk, this leads to discuss the solution geometrically. The upper bound of outcomes is determined by suggesting a majorant analytic function in $U$ (for two functions characterized by a power series, a majorant is the summation of a power series with positive coefficients which are not less than the absolute values of the conforming coefficients of the assumed series). This technique is very useful in approximation theory.

Received 2019-01-13; accepted 2019-02-25; published 2019-05-01.

2010 Mathematics Subject Classification. 34A12,45C30.

Key words and phrases. fractional calculus; fractional differential equation; fractional operator.

(C)2019 Authors retain the copyrights of their papers, and all open access articles are distributed under the terms of the Creative Commons Attribution License. 


\section{IntroduCtion}

Time scales calculus [1] cards us to teaching the dynamic equations, which contains both differences and differential equations, both of which are substantial in understanding applications. The dynamical behavior of different classes of fractional operating formals on time scales is presently experiencing active studies. Several authors considered the existence and uniqueness solutions for problems involving classical fractional derivative (see [2]- [10]). Holomorphic solution for some complex fractional classes is given in [5]- [7]. In this work, we use a majorant technique of analytic functions to prove the convergent of outcomes. We generalize some properties by applying the concept of classic fractional derivative formal operator.

Our construction is furnished by the Riemann-Liouville fractional operators.

Definition 1. The Riemann-Liouville fractional integral formal of the function $\phi$ of arbitrary order $\alpha>0$ is given by

$$
I_{a}^{\alpha} \phi(\wp)=\int_{a}^{\wp} \frac{(\wp-\tau)^{\alpha-1}}{\Gamma(\alpha)} \phi(\tau) d \tau
$$

Definition 2. The Riemann-Liouville fractional differential formal of the function $\phi$ of arbitrary order $\alpha \in[0,1)$ is given by

$$
D_{a}^{\alpha} \phi(\wp)=\frac{d}{d \wp} \int_{a}^{\wp} \frac{(\wp-\tau)^{-\alpha}}{\Gamma(1-\alpha)} \phi(\tau) d \tau=\frac{d}{d \wp} I_{a}^{1-\alpha} \phi(\wp)
$$

Definition 3. [8] The majorant formula is given by : $\sigma(\chi)=\sum \sigma_{i} \chi^{i}$ and $\Lambda(\chi)=\sum \Lambda_{i} \chi^{i}$, then $\sigma(\chi) \ll \Lambda(\chi)$ if and only if $\left|\sigma_{i}\right| \leq\left|\Lambda_{i}\right|$ for each $i$. Similarly, if

$$
\rho(\wp, \chi)=\sum \rho_{i k}(\wp-\varepsilon)^{i} \chi^{k}
$$

and

$$
\Theta(t, \chi)=\sum \Theta_{i k}(\wp-\varepsilon)^{i} \chi^{k}
$$

then $\rho(\wp, \chi) \ll_{\varepsilon} \Theta(\wp, \chi)$ if and only if $\left|\rho_{i k}\right| \leq \Theta_{i k}$ for all $i$ and $k$.

Define the family of majorant functions: for each $k \in \mathbb{N}$, we set

$$
\Xi_{\nu}^{(k)}(z)=\sum_{n=0}^{\infty} \frac{z^{n}}{\nu(n+1)^{k+2}}, \quad(|z|<1, \nu \geq 1)
$$

Clearly that for every $k \in \mathbb{N}, \nu \geq 1$, the functional $\Xi_{\nu}^{(k)}$ converges for all values $|z|<1$. Further, this functional has some significant majorant correlations as follows: 
Proposition 1. The following inequalities hold.

(i) $\Xi_{\nu}^{(0)}(z) \Xi_{\nu}^{(0)}(z) \ll \Xi_{\nu}^{(0)}(z)$;

(ii) $\Xi_{\nu}^{(0)}(z) \gg \Xi_{\nu}^{(1)}(z) \gg \Xi_{\nu}^{(2)}(z) \gg \cdots ;$

(iii) $\frac{1}{2^{i+2}} \Xi_{\nu}^{(i-1)}(z) \ll \frac{d}{d z} \Xi_{\nu}^{(k)}(z) \ll \Xi_{\nu}^{(k-1)}(z)$ and

$\frac{1}{2^{k+2}} \Xi_{\nu}^{(k-2)}(z) \ll \frac{d^{2}}{d z^{2}} \Xi_{\nu}^{(k)}(z) \ll \Xi_{\nu}^{(k-2)}(z) ;$

(iv) $\Xi_{\nu}^{(k)}(z) \Xi_{\nu}^{(k)}(z) \cdots \Xi_{\nu}^{(k)}(z) \ll \Xi_{\nu}^{(k)}(z)$;

(v) $\frac{1}{1-\varepsilon z} \Xi_{\nu}^{(k)}(z) \ll C_{i, \varepsilon} \Xi_{\nu}^{(k)}(z)$,

$\left(\varepsilon \in(0,1)\right.$, and $\left.C_{k, \varepsilon} \in(0, \infty)\right)$;

(vi) $\frac{\Xi_{\nu}^{(k-2)}(z)}{(2 \nu)^{3(k+2)}} \ll D_{a}^{\alpha} \Xi_{\nu}^{(k)}(z)$,

for sufficient large $\nu \geq 1$.

Proof. By employing the formula expansion of $\Xi_{\nu}^{(k)}(z)$, inequalities (i) and (ii) are achieved. According to the following inequalities:

$$
\begin{aligned}
\frac{1}{\nu 2^{k+2}(n+1)^{k+1}} & =\frac{n+1}{\nu 2^{k+2}(n+1)^{k+2}} \\
& =\frac{n+1}{\nu(2 n+2)^{k+2}} \\
& \leq \frac{n+1}{\nu(n+2)^{k+2}}
\end{aligned}
$$

and

$$
\begin{aligned}
\frac{n+1}{\nu(n+2)^{k+2}} & <\frac{n+2}{\nu(n+2)^{k+2}} \\
& =\frac{1}{\nu(n+2)^{k+1}} \\
& \leq \frac{1}{\nu(n+1)^{k+1}},
\end{aligned}
$$

we get (iii). Similarly for (iv). To show (v), by arbitrary choice of $\varepsilon$, we assume that

$$
\varepsilon^{n} \leq \frac{C_{k, \varepsilon}}{\nu(n+1)^{k+2}}
$$

which leads to

$$
\frac{1}{1-\varepsilon z}=\sum_{n=0}^{\infty} \varepsilon^{n} z^{n} \ll C_{i, \varepsilon} \Xi_{\nu}^{(k)}(z) .
$$

This implies that for all $n$

$$
\frac{1}{1-\varepsilon z} \Xi_{\nu}^{(k)}(z) \ll C_{i, \varepsilon} \Xi_{\nu}^{(k)}(z)
$$


Finally, by using the relation

$$
D_{a}^{\alpha} \Xi_{\nu}^{(k)}(z)=\sum_{n=0}^{\infty} \frac{\Gamma(n+1)}{\Gamma(n+1-\alpha)(n+1)^{k+2}} z^{n-\alpha}, \quad(|z|<1)
$$

we get inequality (vi) for sufficient large $\nu \geq 1$.

In the same manner of Proposition 1, we have the following result:

Proposition 2. If $\phi(z)$ is holomorphic in a proximity of $|z| \leq r_{0}$, then $\phi(z)$ is majorized by

$$
\phi(z) \ll \frac{M}{1-\left(\frac{z}{r_{0}}\right)^{2}} \ll \frac{M}{1-\left(\frac{\varepsilon z}{r}\right)^{2}} \times \Xi_{\nu}^{(k)}\left(\frac{z}{r}\right) \ll M C_{k, \varepsilon} \Xi_{\nu}^{(k)}\left(\frac{z}{r}\right),
$$

for any $0<r<\varepsilon r_{0}$.

\section{Fractional operator formal}

Assume that $\mathfrak{H}(\wp, z, v, v, w), \wp \in J=[\mathfrak{a}, T]$ is a holomorphic function in a proximity of the four dim. point $(a, b, c, d, e) \in J \times \mathbb{C}^{4}$, and suppose that $\psi(z)$ is a holomorphic function in a proximity of $z=b$ achieving

$$
\psi(b)=c, \frac{\partial \psi}{\partial z}(b)=d \text { and } \frac{\partial^{2} \psi}{\partial z^{2}}(b)=e .
$$

Consider the initial value problem

$$
\left\{\begin{array}{l}
\frac{\partial^{\alpha} v(\wp, z)}{\partial \wp^{\alpha}}=\mathfrak{H}\left(\wp, z, v, \frac{\partial v}{\partial z}, \frac{\partial^{2} v}{\partial z^{2}}\right), \\
v(a, z)=\psi(z), \text { in a proximity of } z=b . \\
(\alpha \in[0,1), z \in U, \wp \in J)
\end{array}\right.
$$

Eq.(2.1) has Cauchy problem when

$$
\mathfrak{H}\left(\wp, z, v, \frac{\partial v}{\partial z}, \frac{\partial^{2} v}{\partial z^{2}}\right) \equiv \Theta\left(\wp, z, v, \frac{\partial v}{\partial z}\right)
$$

and diffusion problem of fractional order when

$$
\mathfrak{H}\left(\wp, z, v, \frac{\partial v}{\partial z}, \frac{\partial^{2} v}{\partial z^{2}}\right) \equiv \Theta\left(\wp, z, v, \frac{\partial^{2} v}{\partial z^{2}}\right) .
$$

Theorem 3. Consider the initial value problem (2.1), then it has a unique holomorphic outcome $(v(\wp, z))$, in a proximity of $(a, b) \in J \times \mathbb{C}$. 
Proof. Move the point $(a, b)$ into the origin $(0,0)$ and change the variable as follows:

$$
\varphi(\wp, z)=v(\wp, z)-\psi(z)
$$

where $\varphi(\wp, z)$ is the new variable, then we get

$$
\left\{\begin{array}{l}
\frac{\partial^{\alpha} \varphi(t, z)}{\partial \wp^{\alpha}}=\Theta\left(\wp, z, \varphi, \frac{\partial \varphi}{\partial z}, \frac{\partial^{2} \varphi}{\partial z^{2}}\right) \\
\varphi(0,0)=0, \text { in a proximity of } z=0 .
\end{array}\right.
$$

Here, the functional $\Theta\left(\wp, z, \varphi, \frac{\partial \varphi}{\partial z}, \frac{\partial^{2} \varphi}{\partial z^{2}}\right)$ is holomorphic in a proximity of the origin in $I \times \mathbb{C}^{4}, \wp \in I=[0,1]$. Therefore, it is sufficient to consider (2.2). Let the above equation has a unique outcome:

$$
\varphi(\wp, z)=\sum_{k=0}^{\infty} \varphi_{k}(z) \wp^{k}, \quad(\wp \in I) .
$$

We show that $\varphi(\wp, z)$ converges.

Let $r_{0}>0$ and $\rho>0$ be small enough and the function $\Theta(\wp, z, \varphi, v, w)$ be holomorphic in a proximity of the set

$$
\mathcal{S}=\left\{(\wp, z, \varphi, v, w) \in I \times \mathbb{C}^{4} ; \wp<\tau \leq 1,|z| \leq r_{0},|\varphi| \leq \rho,|v| \leq \rho \text { and }|w| \leq \rho\right\}
$$

Assume that $\Theta$ is bounded by $M$ in this domain. Since $\Theta$ is holomorphic, then it has the following construction:

$$
\begin{gathered}
\Theta(\wp, z, \varphi, v, w)=\sum_{p, q, s, l} a_{p, q, s, l}(z) \wp^{p} \varphi^{q} v^{s} w^{l}, \\
(\wp \in I,(\varphi, v, w) \in(\mathbb{C} \times \mathbb{C} \times \mathbb{C})) .
\end{gathered}
$$

In virtue of the Cauchy's inequality and the certainty that the coefficient $a_{p, q, s}(z)$ is holomorphic in a proximity of $\left\{z \in \mathbb{C} ;|z| \leq r_{0}\right\}$, implies that

$$
a_{p, q, s, l}(z) \ll \frac{M}{\tau^{p} \rho^{q+s+l}} \frac{1}{1-\left(\frac{z}{r_{0}}\right)^{2}} .
$$

In this case, the problem turns to evaluate a function $\vartheta(\wp, z)$ satisfying the majorant inequalities 


$$
\left\{\begin{array}{l}
\frac{\partial^{\alpha} \vartheta(\wp, z)}{\partial \wp^{\alpha}} \gg \sum_{p, q, s, l} \frac{M}{\tau^{p} \rho^{q+s+l}} \frac{1}{1-\left(\frac{z}{r_{0}}\right)^{2}} \wp^{p} \vartheta^{q}\left(\frac{\partial \vartheta}{\partial z}\right)^{s}\left(\frac{\partial^{2} \vartheta}{\partial z^{2}}\right)^{l}, \quad \wp \in I \\
\vartheta(0,0) \gg 0
\end{array}\right.
$$

then the function $\vartheta(\wp, z)$ majorizes the formal solution $\varphi(\wp, z)$. Assume $0<r<r_{0}$ and define

$$
\vartheta(\wp, z)=L \Xi_{\nu}^{(2)}\left(\wp+\left(\frac{z}{r}\right)^{2}\right),(L>0) .
$$

Operating by the fractional differential formal with respect to $\wp$ we get

$$
\frac{\partial^{\alpha} \vartheta(\wp, z)}{\partial \wp^{\alpha}}=L \frac{\partial^{\alpha} \Xi_{\nu}^{(2)}\left(\wp+\left(\frac{z}{r}\right)^{2}\right)}{\partial \wp^{\alpha}},(L>0) .
$$

Then by Proposition 1 (vi) we get

$$
\frac{\partial^{\alpha} \vartheta(\wp, z)}{\partial \wp^{\alpha}} \gg \frac{L}{C_{\nu}} \Xi_{\nu}^{(0)}\left(\wp+\left(\frac{z}{r}\right)^{2}\right),
$$

where $C_{\nu}:=(2 \nu)^{12}$. For a constant $K_{0}>0$ again in virtue of Proposition 1 (ii) and (iii) we find

$$
\begin{aligned}
& \sum_{p, q, s, l} \frac{M}{\rho^{q+s+l}} \frac{1}{1-\left(\frac{z}{r_{0}}\right)^{2}} \frac{1}{1-\frac{\wp}{\tau}} \vartheta^{q}\left(\frac{\partial \vartheta}{\partial z}\right)^{s}\left(\frac{\partial^{2} \vartheta}{\partial z^{2}}\right)^{l} \\
& \ll \sum_{p, q, s} \frac{M}{\rho^{q+s}} \frac{1}{1-\left(\frac{z}{r_{0}}\right)^{2}-\frac{\wp}{\tau}}\left\{L \Xi_{\nu}^{(2)}\left(\wp+\left(\frac{z}{r}\right)^{2}\right)\right\}^{q} \\
& \times\left\{\frac{2 r_{0} L}{r^{2}} \Xi_{\nu}^{(0)}\left(\wp+\left(\frac{z}{r}\right)^{2}\right)\right\}^{s}\left\{\frac{2 r_{0} L}{r^{2}} \Xi_{\nu}^{(0)}\left(\wp+\left(\frac{z}{r}\right)^{2}\right)\right\}^{l} \\
& \ll \sum_{p, q, s} \frac{M}{\rho^{q+s}} \frac{1}{1-\left(\frac{z}{r_{0}}\right)^{2}-\frac{\wp}{\tau}}\left\{L \Xi_{\nu}^{(0)}\left(\wp+\left(\frac{z}{r}\right)^{2}\right)\right\}^{q} \\
& \times\left\{\frac{2 r_{0} L}{r^{2}} \Xi_{\nu}^{(0)}\left(\wp+\left(\frac{z}{r}\right)^{2}\right)\right\}^{s}\left\{\frac{2 r_{0} L}{r^{2}} \Xi_{\nu}^{(0)}\left(\wp+\left(\frac{z}{r}\right)^{2}\right)\right\}^{l} \\
& \ll \frac{M K_{0}}{1-L / \rho-4 L r_{0} / \rho r^{2}} \Xi_{\nu}^{(0)}\left(\wp+\left(\frac{z}{r}\right)^{2}\right),
\end{aligned}
$$

whenever,

$$
\frac{L}{\rho}+\frac{4 r_{0} L}{r^{2}}<1 .
$$

Comparing (2.7) and (2.8) with the inequality

$$
\frac{L}{C_{\mu}} \geq \frac{M K_{0}}{1-L / \rho-4 L r_{0} / \rho r^{2}}
$$

then we obtain the majorant inequalities in (2.4) are achieved.

Note that relation (2.9) holds by choosing a sufficiently small $L, r_{0}$, such that

$$
\frac{L}{\rho}+\frac{4 r_{0} L}{r^{2}}<1
$$


Hence, $\vartheta(\wp, z)$ in $(2.5)$ majorizes the formal solution $\psi(\wp, z)$. This now implies that $\varphi(\wp, z)$ converges in a domain containing $\left\{(\wp, z) \in I \times \mathbb{C} ;\left|\wp+\left(\frac{z}{r}\right)^{2}\right|<1\right\}$.

\section{Continuation outcomes}

Suppose that $\Omega$ is a proximate of the origin $(0,0)$ and $\mathfrak{H}(\wp, z, v, v, w), \wp \in I$, is a holomorphic function in $\Omega \times \mathbb{C}_{v} \times \mathbb{C}_{v} \times \mathbb{C}_{w}$. Consider the following equation:

$$
\frac{\partial^{\alpha} v}{\partial \wp^{\alpha}}=\mathfrak{H}\left(\wp, z, v, \frac{\partial v}{\partial z}, \frac{\partial^{2} v}{\partial z^{2}}\right)
$$

Then we have

$$
\mathfrak{H}(\wp, z, v, v)=\sum_{j, p, q} a_{j, p, q}(\wp, z) v^{j} v^{p} w^{q} .
$$

Define the following two sets:

$$
S_{0}=\left\{(j, p, q) \in \mathbb{N}^{3} ; a_{j, p, q}(\wp, z) \neq 0\right\}
$$

and

$$
S=\left\{(j, p, q) \in S_{0} ; j+p+q \geq 3\right\}
$$

Clearly, $\mathfrak{H}$ is linear if and only if $S=\emptyset$; and it is nonlinear otherwise.

Suppose that $\mathfrak{H}$ is nonlinear, this implies that $S$ is nonempty with the coefficients formal

$$
a_{j, p, q}(\wp, z)=\wp^{k_{j, p, q}} b_{j, p, q}(\wp, z)
$$

where $k_{j, p, q}$ is a non-negative integer and $b_{j, p, q}(0, z)=0$. Applying $(3.1)$, we get

$$
\frac{\partial^{\alpha} v}{\partial \wp^{\alpha}}=\sum_{j, p, q} \wp^{k_{j, p, q}} b_{j, p, q}(\wp, z) v^{j}\left(\frac{\partial v}{\partial z}\right)^{p}\left(\frac{\partial^{2} v}{\partial z^{2}}\right)^{q}
$$

For $\kappa \in \mathbb{R}$, we have

$$
\delta(\kappa):=\inf _{(j, p, q) \in S}\left(k_{j, p, q}+1+\kappa(j+p+q-1)\right) .
$$

It is clear that, when $\kappa=0$, we get $\delta(\kappa) \geq 1$. Also, if

$$
\kappa>\sup _{(j, p, q) \in S}\left|\frac{\left(k_{j, p, q}+1\right)}{j+p+q-1}\right|,
$$

then $\delta(\kappa)$ is positive.

Next, we show that $v(\wp, z)=O\left(\wp^{\kappa}\right)$ is analytically continued up to a proximate of the origin. 
Theorem 4. Let $v(\wp, z)$ be a holomorphic solution of (3.4) in $\Omega$. Then for some $\kappa \in \mathbb{R}$ achieving $\delta(\kappa)>0$, such that

$$
\sup _{z \in \mathbb{C}}|v(\wp, z)|=O\left(\wp^{\kappa}\right), \quad(\wp \rightarrow 0),
$$

and the solution $v(\wp, z)$ can be expanded analytically as a holomorphic outcome of Eq. (3.4) up to a approximate of the origin.

Proof. Suppose that $v(\wp, z)$ is a solution for Eq. (3.4), which is holomorphic in the domain $\Omega$. In addition, we let the expansion (3.2) is valid in the domain $\Delta$ where

$$
\Delta:=\{(\wp, z, v, v, w): \wp \leq 2 \tau,|z| \leq 2 r,|v| \leq \rho,|v| \leq \rho,|w| \leq \rho\},
$$

such that $\tau<1,2 r<1$ and $\rho$ is positive number. Let $M$ be a bound of $\mathfrak{F}$ in $\Delta$.

Now we suggest the following initial value problem in

$$
\begin{gathered}
\chi(\wp, z):=\sum_{k=0}^{\infty} \chi_{k}(z)(\wp-\varepsilon)^{k}: \\
\left\{\begin{array}{l}
\frac{\partial^{\alpha} \chi(\wp, z)}{\partial \wp^{\alpha}}=\sum_{j, p, q} \wp^{k_{j, p, q}} b_{j, p, q}(\wp, z) \chi^{j}\left(\frac{\partial \chi}{\partial z}\right)^{p}\left(\frac{\partial^{2} \chi}{\partial z^{2}}\right)^{q}, \quad \wp \in I \\
\chi(\varepsilon, z)=v(\varepsilon, z) .
\end{array}\right.
\end{gathered}
$$

We aim to show that the formal $\chi(\wp, z)$ converges in a domain including the origin. This leads to $v(\wp, z)$ can be continued analytically by $\chi(\wp, z)$ up to some neighborhood of the origin.

But $v(\wp, z)=O\left(\wp^{\kappa}\right)$ as $\wp \rightarrow 0$, this implies that there exists a positive constant $C_{0}>0$ such that $|u(\varepsilon, z)| \leq$ $C_{0} \varepsilon^{\kappa}$ uniformly in $z$. Thus, by Proposition 2 , for some positive constant $C_{1}>0$, we get

$$
v(\varepsilon, z) \ll C_{0} \varepsilon^{\kappa} C_{1} \Xi_{\nu}^{(2)}\left(\frac{\wp-\varepsilon}{c \tau}+\left(\frac{z}{r}\right)^{2}\right) .
$$

Assume that $0<\kappa<1$ (without lose generality). To formulate an inequality satisfying the majorant function, we majorize the expression $\wp^{k_{j, p}} b_{j, p}(\wp, z)$ by using $\Xi_{\nu}^{(0)}(z)$. Let

$$
\Lambda:=\left(\frac{z}{r}\right)^{2}+\frac{\wp-\varepsilon}{c \tau}
$$

then $\wp$ is majorized by

$$
\begin{aligned}
\wp & =\varepsilon+(\wp-\varepsilon) \ll_{\varepsilon}(\varepsilon+4 c \tau)\left(1+\frac{\wp-\varepsilon}{4 c \tau}\right) \\
& \ll_{\varepsilon}(\varepsilon+4 c \tau) \Xi_{\nu}^{(0)}(\Lambda) .
\end{aligned}
$$

We proceed to extend the function $b_{j, p, q}(\wp, z)$ as follows 


$$
b_{j, p, q}(\wp, z)=\sum_{m=0}^{\infty} b_{j, p, q}^{(m)}(z) \wp^{m}
$$

where each $b_{j, p, q}^{(m)}$ is holomorphic in some domain of $\{|z| \leq 2 r\}$ and achieves

$$
\left|b_{j, p, q}^{(m)}(z)\right| \leq \frac{M}{\rho^{j+p+q}(2 \tau)^{m+k_{j, p, q}}} .
$$

This estimate poses,

$$
b_{j, p, q}^{(m)}(z) \ll \frac{C_{0} C_{1} \Xi_{\nu}^{(0)}(\Lambda)}{\rho^{j+p+q}(2 \tau)^{m+k_{j, p, q}}}
$$

where $C_{1}$ is a positive constant satisfying (3.7). Joining relations (3.8) and (3.9) and using Proposition 1 (i), we get

$$
\begin{aligned}
\wp^{k_{j, p, q}} b_{j, p, q}(\wp, z) & \ll_{\varepsilon} \sum_{m=0}^{\infty}\left[(\varepsilon+4 c \tau) \Xi_{\nu}^{(0)}(\Lambda)\right]^{m+k_{j, p, q}}\left[\frac{C_{0} C_{1} \Xi_{\nu}^{(0)}(\Lambda)}{\rho^{j+p+q}(2 \tau)^{m+k_{j, p, q}}}\right] \\
& \ll_{\varepsilon} \frac{C_{0} C_{1}}{\rho^{j+p+q}} \Xi_{\nu}^{(0)}(\Lambda) \sum_{m=0}^{\infty}(4 c)^{m+k_{j, p, q}} .
\end{aligned}
$$

Putting $\varepsilon=\frac{c \tau}{2}$ and $0<c \leq 1$ and fixing $r$ so that $c r<1 / 4,0<c \leq 1$, we finally get the formal

$$
\wp^{k_{j, p, q}} b_{j, p, q}(\wp, z) \ll_{\varepsilon} \frac{2 C_{0} C_{1}}{\rho^{j+p+q}} \Xi_{\nu}^{(0)}(\Lambda) .
$$

Therefore, the function $W(\wp, z)$ achieves the majorant conclusion

$$
\left\{\begin{array}{l}
\frac{\partial^{\alpha} W}{\partial \wp^{\alpha}} \gg_{\varepsilon} \sum_{j, p, q} \frac{2 C_{0} C_{1}}{\rho^{j+p+q}} \Xi_{\nu}^{(0)}(\Lambda) W^{j}\left(\frac{\partial W}{\partial \Lambda}\right)^{p}\left(\frac{\partial^{2} W}{\partial \Lambda^{2}}\right)^{q}, \quad \wp \in I \\
W(\varepsilon, z) \gg_{\varepsilon} \varepsilon^{\kappa} C_{0} C_{1} \Xi_{\nu}^{(2)}(\Lambda)
\end{array}\right.
$$

is one majorant function for the formal solution $\chi(\wp, z)$. In the similar manner of the proof of Theorem 3 by choosing suitable values for $\rho>0, c>0$, and letting $\varepsilon=\frac{c \tau}{2}$, we have

$$
W(\wp, z)=\varepsilon^{\kappa} C_{0} C_{1} \Xi_{\nu}^{(2)}(\Lambda)
$$

achieves the majorant formal given in (3.11). Thus, $W(\wp, z)$ is holomorphic in a domain involving $(0,0)$; consequently must be true for $\chi(\wp, z)$.

\section{Acknowledgments}

The authors would like to express their thanks to the reviewers for their important and useful comments to improve the paper. The work here is partially supported by the Universiti Kebangsaan Malaysia grant: GUP (Geran Universiti Penyelidikan)-2017-064. 


\section{REFERENCES}

[1] M. Bohner, A. Peterson, Dynamic equations on times scale, Birkhauser Boston, Boston, MA, 2001.

[2] A. A. Kilbas, H. M. Srivastava and J.J. Trujillo, Theory and applications of fractional differential equations. North-Holland, Mathematics Studies, Elsevier 2006.

[3] J. Sabatier, O. P. Agrawal, J. A. Tenreiro Machado, Advance in Fractional Calculus: Theoretical Developments and Applications in Physics and Engineering, Springer, 2007.

[4] D. Baleanu, B. Guvenc Ziya, J. A. Tenreiro, New Trends in Nanotechnology and Fractional Calculus Applications, Springer, 2009.

[5] R. W. Ibrahim, Existence and uniqueness of holomorphic solutions for fractional Cauchy problem, J. Math. Anal. Appl. 380 (2011), 232-240.

[6] R. W. Ibrahim, On holomorphic solutions for nonlinear singular fractional differential equations, Comput. Math. Appl. 62 (3) (2011), 1084-1090.

[7] R. W. Ibrahim, Generalized Ulam-Hyers stability for fractional differential equations, Int. J. Math. 23 (5) (2012), Art. ID 1250056.

[8] R. W. Ibrahim, The fractional differential polynomial neural network for approximation of functions, Entropy 15 (10) (2013), 4188-4198.

[9] R. W. Ibrahim, et al., Fractional differential texture descriptors based on the Machado entropy for image splicing detection. Entropy 17 (7) (2015), 4775-4785.

[10] R. W. Ibrahim and M. Darus, Analytic Study of Complex Fractional Tsallis Entropy with Applications in CNNs, Entropy 20 (10) (2018), Art. ID 722. 